\title{
Correlations and Equilibration in Relativistic Quantum Systems ${ }^{\star}$
}

\author{
W. Cassing and S. Juchem \\ Institut für Theoretische Physik, Universität Giessen, Heinrich-Buff-Ring 16, D-35392 \\ Giessen, Germany
}

\begin{abstract}
In this article we study the time evolution of an interacting field theoretical system, i.e. $\phi^{4}$-field theory in $2+1$ space-time dimensions, on the basis of the KadanoffBaym equations for a spatially homogeneous system including the self-consistent tadpole and sunset self-energies. We find that equilibration is achieved only by inclusion of the sunset self-energy. Simultaneously, the time evolution of the scalar particle spectral function is studied for various initial states. We also compare associated solutions of the corresponding Boltzmann equation to the full Kadanoff-Baym theory. This comparison shows that a consistent inclusion of the spectral function has a significant impact on the equilibration rates only if the width of the spectral function becomes larger than $1 / 3$ of the particle mass. Furthermore, based on these findings, the conventional transport of particles in the on-shell quasiparticle limit is extended to particles of finite life time by means of a dynamical spectral function $A\left(X, \boldsymbol{p}, M^{2}\right)$. The off-shell propagation is implemented in the Hadron-String-Dynamics (HSD) transport code and applied to the dynamics of nucleus-nucleus collisions.
\end{abstract}

\section{Introduction}

The many-body theory of strongly interacting particles out of equilibrium is a challenging problem since a couple of decades. Many approaches based on the Martin-Schwinger hierarchy of Green's functions [1] have been formulated [2-6] and applied to model cases. Nowadays, the dynamical description of strongly interacting systems out of equilibrium is dominantly based on on-shell transport theories and efficient numerical recipies have been set up for the solution of the coupled channel transport equations [7-13] (and Refs. therein). These transport approaches have been derived either from the Kadanoff-Baym equations [14] in Refs. [15-19] or from the hierarchy of connected equal-time Green's functions $[2,20]$ in Refs. $[3,10,21]$ by applying a Wigner transformation and restricting to first order in the derivatives of the phase-space variables $(X, p)$.

However, as recognized early in these derivations [3,17], the on-shell quasiparticle limit, that invoked additionally a reduction of the $8 N$-dimensional phasespace to $7 N$ independent degrees of freedom, where $N$ denotes the number of particles in the system, should not be adequate for particles of short life time and/or high collision rates. Therefore, transport formulations for quasiparticles with dynamical spectral functions have been presented in the past [18] providing a formal basis for an extension of the presently applied transport models.

^ supported by GSI Darmstadt 
Apart from the transport extensions mentioned above there is a further branch discussing the effects from non-local collisions terms (cf. Refs. [22-27]).

The basic questions in this field are:

i) What are the microscopic mechanisms that lead to thermalization though the underlying quantum theory is time reversible?

ii) What are the effects of off-shell transitions in case of strong coupling?

iii) Is there an adequate semiclassical limit to the full quantum theory?

In this article we will attempt to shed some light on these questions for fully relativistic self-interacting systems and review some recent progress achieved in the last years. Our work is organized as follows: In Section 2 we will study numerically the self-interacting scalar $\phi^{4}$-theory in $2+1$ dimensions on the basis of the Kadanoff-Baym equations for homogeneous systems and concentrate on the time evolution of correlations and the single-particle spectral function. A comparison to the respective Boltzmann limit is performed in Section 3. Furthermore, the derivation of a semiclassical off-shell transport theory for inhomogeneous systems is presented in Section 4 with special emphasis on the time evolution of spectral functions.

\section{The Kadanoff-Baym Equations}

We briefly recall the basic equations for Green's functions and particle selfenergies as well as their symmetry properties that will be exploited throughout this work.

\subsection{Preliminaries}

Within the framework of the closed-time-path formalism [1,14] Green's functions $G$ and self-energies $\Sigma$ are given as path ordered quantities. They are defined on the time contour consisting of two branches from $(+)-\infty$ to $\infty$ and $(-)$ from $\infty$ to $-\infty$. For convenience these propagators and self-energies are transformed into a $2 \times 2$ matrix representation according to their path structure [4], i.e. according to the chronological $(+)$ or antichronological $(-)$ branch for the time coordinates $x_{0}$ and $y_{0}$. Explicitly the Green's functions are given by

$$
\begin{aligned}
& i G_{x y}^{c}=i G_{x y}^{++}=\left\langle T^{c}\left\{\phi(x) \phi^{\dagger}(y)\right\}\right\rangle, \\
& i G_{x y}^{<}=i G_{x y}^{+-}=\eta\left\langle\left\{\phi^{\dagger}(y) \phi(x)\right\}\right\rangle, \\
& i G_{x y}^{>}=i G_{x y}^{-+}=\left\langle\left\{\phi(x) \phi^{\dagger}(y)\right\}\right\rangle, \\
& i G_{x y}^{a}=i G_{x y}^{--}=\left\langle T^{a}\left\{\phi(x) \phi^{\dagger}(y)\right\}\right\rangle .
\end{aligned}
$$

where the subscript $\cdot_{x y}$ denotes the dependence on the coordinate space variables $x$ and $y$ and $T^{c}\left(T^{a}\right)$ represent the (anti-)time-ordering operators. In the definition of $G^{<}$the factor $\eta=+1$ stands for bosons and $\eta=-1$ for fermions. In the following we will consider a theory for scalar bosons. The full Green's 
functions are determined via the Dyson-Schwinger equations for path-ordered quantities, here given in $2 \times 2$ matrix representation as

$$
\left(\begin{array}{cc}
G^{c} & G^{<} \\
G^{>} & G^{a}
\end{array}\right)_{x y}=\left(\begin{array}{cc}
G_{o}^{c} & G_{o}^{<} \\
G_{o}^{>} & G_{o}^{a}
\end{array}\right)_{x y}+\left(\begin{array}{cc}
G_{o}^{c} & G_{o}^{<} \\
G_{o}^{>} & G_{o}^{a}
\end{array}\right)_{x z} \odot\left(\begin{array}{cc}
\Sigma^{c} & -\Sigma^{<} \\
-\Sigma^{>} & \Sigma^{a}
\end{array}\right)_{z z^{\prime}} \odot\left(\begin{array}{cc}
G^{c} & G^{<} \\
G^{>} & G^{a}
\end{array}\right)_{z^{\prime} y}(2)
$$

The self-energies $\Sigma^{\cdot}$ are also defined according to their time structure while the symbol ' $\odot$ ' implies an integration over the intermediate space-time coordinates from $-\infty$ to $\infty$. Linear combinations of diagonal and off-diagonal matrix elements give the retarded and advanced Green's functions $G^{\text {ret/adv }}$ and selfenergies $\Sigma^{\text {ret/adv }}$

$$
\begin{array}{ll}
G_{x y}^{\mathrm{ret}}=G_{x y}^{c}-G_{x y}^{<}=G_{x y}^{>}-G_{x y}^{a}, & G_{x y}^{\mathrm{adv}}=G_{x y}^{c}-G_{x y}^{>}=G_{x y}^{<}-G_{x y}^{a}, \\
\Sigma_{x y}^{\mathrm{ret}}=\Sigma_{x y}^{c}-\Sigma_{x y}^{<}=\Sigma_{x y}^{>}-\Sigma_{x y}^{a}, & \Sigma_{x y}^{\mathrm{adv}}=\Sigma_{x y}^{c}-\Sigma_{x y}^{>}=\Sigma_{x y}^{<}-\Sigma_{x y}^{a} .
\end{array}
$$

Resorting equations (2) one obtains Dyson-Schwinger equations for the retarded (advanced) Green's functions (where only the respective self-energies are involved)

$$
\hat{G}_{o, x}^{-1} G_{x y}^{\mathrm{ret} / \mathrm{adv}}=\delta_{x y}+\Sigma_{x z}^{\mathrm{ret} / \mathrm{adv}} \odot G_{z y}^{\mathrm{ret} / \mathrm{adv}},
$$

and the wellknown Kadanoff-Baym equation for the Wightman function $G^{<}$,

$$
\hat{G}_{o, x}^{-1} G_{x y}^{<}=\Sigma_{x z}^{\mathrm{ret}} \odot G_{z y}^{<}+\Sigma_{x z}^{<} \odot G_{z y}^{\mathrm{adv}} .
$$

In these equations $\hat{G}_{o, x}^{-1}$ denotes the (negative) Klein-Gordon differential operator which for bosonic field quanta of mass $m$ is given by $\hat{G}_{o, x}^{-1}=-\left(\partial_{x}^{\mu} \partial_{\mu}^{x}+m^{2}\right)$. The Klein-Gordon equation is solved by the free propagators $G_{o}$ as

$$
\hat{G}_{o, x}^{-1}\left(\begin{array}{cc}
G_{o}^{c} & G_{o}^{<} \\
G_{o}^{>} & G_{o}^{a}
\end{array}\right)_{x y}=\delta_{x y}\left(\begin{array}{cc}
1 & 0 \\
0 & -1
\end{array}\right), \quad \hat{G}_{o, x}^{-1} \quad G_{o, x y}^{\mathrm{ret} / \mathrm{adv}}=\delta_{x y}
$$

with the $\delta$-distribution $\delta_{x y} \equiv \delta^{(d+1)}(x-y)$ for $d+1$ space-time dimensions.

\subsection{Equilibration Within the Scalar $\phi^{4}$-Theory}

The scalar $\phi^{4}$-theory is an example for a fully relativistic field theory of interacting scalar particles that allows to test theoretical approximations without coming to the problems of gauge invariant truncation schemes [28-31]. Its Lagrangian density is given by $(x=(t, \boldsymbol{x}))$

$$
\mathcal{L}(x)=\frac{1}{2} \partial_{\mu} \phi(x) \partial^{\mu} \phi(x)-\frac{1}{2} m^{2} \phi^{2}(x)-\frac{\lambda}{4 !} \phi^{4}(x),
$$

where $m$ denotes the mass and $\lambda$ is the coupling strength determining the interaction strength of the scalar fields. Its quantization is performed in the canonical form (in $d$ spatial dimensions)

$$
\left[\phi(x), \partial_{y_{0}} \phi(y)\right]_{x_{0}=y_{0}}=i \delta^{(d)}(\boldsymbol{x}-\boldsymbol{y}) .
$$


Since for the real boson theory (7) the relation $G^{>}(x, y)=G^{<}(y, x)$ holds (1) the knowledge of the Green's functions $G^{<}(x, y)$ for all $x, y$ characterizes the system completely. Nevertheless, we will give the equations for $G^{<}$and $G^{>}$ explicitly since this is the familiar representation for general field theories [5]. Self-consistent equations of motion are obtained by a loop expansion of the twoparticle irreducible (2PI) effective action $\Gamma$ on the closed time path. It is given by the Legendre transform of the generating functional of the connected Green's functions $W$ as

$$
\Gamma[G]=\Gamma^{o}+\frac{i}{2}\left[\ln \left(1-\odot_{p} G_{o} \odot_{p} \Sigma\right)+\odot_{p} G \odot_{p} \Sigma\right]+\Phi[G] .
$$

in case of vanishing vacuum expectation value $\langle 0|\phi(x)| 0\rangle=0$ [32]. In (9) $\Gamma^{o}$ depends only on free Green's functions and is treated as a constant while the symbols $\odot_{p}$ represent convolution integrals over the closed time path. The functional $\Phi$ is the sum of all closed $2 P I$ diagrams built up by full propagators $G$; it determines the self-energies by functional variation as

$$
\Sigma(x, y)=2 i \frac{\delta \Phi}{\delta G(y, x)} .
$$

Taking into account contributions up to the 3-loop order the $\Phi$ functional reads explicitly for the problem (7)

$$
i \Phi=\frac{i \lambda}{8} \int_{\mathcal{C}} d^{d+1} x G(x, x)^{2}-\frac{\lambda^{2}}{48} \int_{\mathcal{C}} d^{d+1} x \int_{\mathcal{C}} d^{d+1} y G(x, y)^{4} .
$$

By using the stationarity condition of the action $\delta \Gamma / \delta G=0$ and resolving the time structure of the path ordered quantities we obtain the Kadanoff-Baym equations for the time evolution of the Wightman function [5,29]:

$$
\begin{aligned}
-\left[\partial_{\mu}^{x} \partial_{x}^{\mu}+m^{2}\right] G^{<}(x, y) & =\Sigma_{\operatorname{tad}}(x) G^{<}(x, y) \\
& +\int_{t_{o}}^{x_{o}} d z_{o} \int d^{d} z\left[\Sigma^{>}(x, z)-\Sigma^{<}(x, z)\right] G^{<}(z, y) \\
& -\int_{t_{o}}^{y_{o}} d z_{o} \int d^{d} z \Sigma^{<}(x, z)\left[G^{>}(z, y)-G^{<}(z, y)\right], \\
-\left[\partial_{\mu}^{y} \partial_{y}^{\mu}+m^{2}\right] G^{<}(x, y)= & \Sigma_{\operatorname{tad}}(y) G^{<}(x, y) \\
& +\int_{t_{o}}^{x_{o}} d z_{o} \int d^{d} z\left[G^{>}(x, z)-G^{<}(x, z)\right] \Sigma^{<}(z, y) \\
& -\int_{t_{o}}^{y_{o}} d z_{o} \int d^{d} z G^{<}(x, z)\left[\Sigma^{>}(z, y)-\Sigma^{<}(z, y)\right],
\end{aligned}
$$

where $d$ denotes the spatial dimension of the problem $(d=2$ in the case considered below). Within the 3-loop approximation for the effective action (i.e. the $\Phi$ 

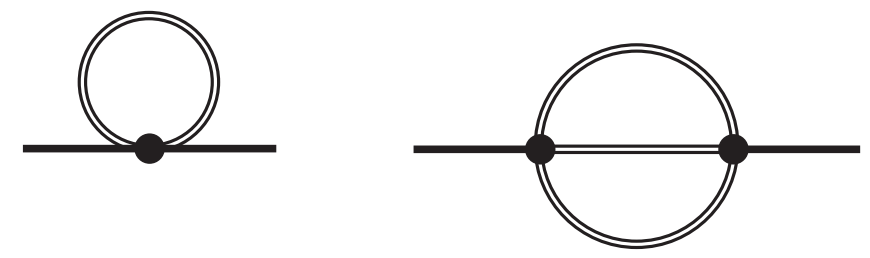

Fig. 1. Self-energies of the Kadanoff-Baym equation: tadpole self-energy (l.h.s.) and sunset self-energy (r.h.s.). Since the lines represent full Green's functions the selfenergies are self-consistent (see text)

functional (11)) we get two different self-energies: In leading order of the coupling constant only the tadpole diagram (l.h.s. of Fig. 1) contributes and leads to the generation of an effective mass for the field quanta. This self-energy (in coordinate space) is given by

$$
\Sigma_{\operatorname{tad}}(x)=\frac{\lambda}{2} i G^{<}(x, x),
$$

and is local in space and time. In next order in the coupling constant (i.e. $\lambda^{2}$ ) the non-local sunset self-energy (r.h.s. of Fig. 1) enters the time evolution as

$$
\begin{aligned}
& \Sigma^{</>}(x, y)=-\frac{\lambda^{2}}{6} G^{</>}(x, y) G^{</>}(x, y) G^{>/<}(y, x) \\
& \longrightarrow \quad \Sigma^{</>}(x, y)=-\frac{\lambda^{2}}{6}\left[G^{</>}(x, y)\right]^{3} .
\end{aligned}
$$

Thus the Kadanoff-Baym equation (16) in our case includes the influence of a mean-field on the particle propagation - generated by the tadpole diagram - as well as of scattering processes as inherent in the sunset diagram.

The Kadanoff-Baym equation describes the full quantum nonequilibrium time evolution on the two-point level for a system prepared at an initial time $t_{0}$, i.e. when higher order correlations are discarded. The causal structure of this initial value problem is obvious since the time integrations are performed over the past up to the actual time $x_{0}$ (or $y_{0}$, respectively) and do not extend to the future.

In the following we will restrict to homogeneous systems in space. To obtain a numerical solution of the Kadanoff-Baym equation (12) is transformed to momentum space:

$$
\begin{aligned}
\partial_{t_{1}}^{2} G^{<}\left(\boldsymbol{p}, t_{1}, t_{2}\right) & =-\left[\boldsymbol{p}^{2}+m^{2}+\bar{\Sigma}_{\mathrm{tad}}\left(t_{1}\right)\right] G^{<}\left(\boldsymbol{p}, t_{1}, t_{2}\right) \\
& -\int_{t_{0}}^{t_{1}} d t^{\prime}\left[\Sigma^{>}\left(\boldsymbol{p}, t_{1}, t^{\prime}\right)-\Sigma^{<}\left(\boldsymbol{p}, t_{1}, t^{\prime}\right)\right] G^{<}\left(\boldsymbol{p}, t^{\prime}, t_{2}\right) \\
& +\int_{t_{0}}^{t_{2}} d t^{\prime} \Sigma^{<}\left(\boldsymbol{p}, t_{1}, t^{\prime}\right)\left[G^{>}\left(\boldsymbol{p}, t^{\prime}, t_{2}\right)-G^{<}\left(\boldsymbol{p}, t^{\prime}, t_{2}\right)\right]
\end{aligned}
$$




$$
=-\left[\boldsymbol{p}^{2}+m^{2}+\bar{\Sigma}_{\mathrm{tad}}\left(t_{1}\right)\right] G^{<}\left(\boldsymbol{p}, t_{1}, t_{2}\right)+I_{1}^{<}\left(\boldsymbol{p}, t_{1}, t_{2}\right)
$$

where we have summarized both memory integrals into the function $I_{1}^{<}$. The equation of motion in the second time direction $t_{2}$ is given analogously. In twotime and momentum space $\left(\boldsymbol{p}, t, t^{\prime}\right)$ representation the self-energies read

$$
\begin{aligned}
\bar{\Sigma}_{\mathrm{tad}}(t) & =\frac{\lambda}{2} \int \frac{d^{d} p}{(2 \pi)^{d}} i G^{<}(\boldsymbol{p}, t, t) \\
\Sigma^{<}\left(\boldsymbol{p}, t, t^{\prime}\right) & =-\frac{\lambda^{2}}{6} \int \frac{d^{d} q}{(2 \pi)^{d}} \int \frac{d^{d} r}{(2 \pi)^{d}} G^{<}\left(\boldsymbol{q}, t, t^{\prime}\right) G^{<}\left(\boldsymbol{r}, t, t^{\prime}\right) G^{>}\left(\boldsymbol{q}+\boldsymbol{r}-\boldsymbol{p}, t^{\prime}, t\right) . \\
& =-\frac{\lambda^{2}}{6} \int \frac{d^{d} q}{(2 \pi)^{d}} \int \frac{d^{d} r}{(2 \pi)^{d}} G^{<}\left(\boldsymbol{q}, t, t^{\prime}\right) G^{<}\left(\boldsymbol{r}, t, t^{\prime}\right) G^{<}\left(\boldsymbol{p}-\boldsymbol{q}-\boldsymbol{r}, t, t^{\prime}\right) .
\end{aligned}
$$

\subsection{Renormalization}

In $2+1$ space-time dimensions both self-energies incorporated (1) are ultraviolet divergent. Since we consider particles with a finite mass no problems arise from the infrared momentum regime. For the renormalization of the divergences we only suppose that the time-dependent nonequilibrium distribution functions are decreasing for large momenta comparable to the equilibrium ones, i.e exponentially. Thus we can apply the usual finite temperature renormalization scheme. By separating the real time (equilibrium) Green's functions into vacuum $(T=0)$ and thermal parts it becomes apparent that only the pure vacuum contributions of the self-energies are divergent. For the linear divergent tadpole diagram we introduce a mass counterterm (at renormalized mass $m$ ) as

$$
\delta m_{\mathrm{tad}}^{2}=\int \frac{d^{2} p}{(2 \pi)^{2}} \frac{1}{2 \omega_{\boldsymbol{p}}}, \quad \omega_{\boldsymbol{p}}=\sqrt{\boldsymbol{p}^{2}+m^{2}},
$$

that cancels the contribution from the momentum integration of the vacuum part of the Green's function. In case of the sunset diagram only the logarithmically divergent pure vacuum part requires renormalization, while it remains finite as long as at least one temperature line is involved. Contrary to the case of $3+1$ dimensions it is not necessary to employ the involved techniques developed for the renormalization of self-consistent theories (in equilibrium) in Refs. [33]. Since the divergence only appears (in energy-momentum space) in the real part of the Feynman self-energy $\Sigma^{c}$ at $T=0$ (and equivalently in the real part of the retarded/advanced self-energies $\left.\Sigma^{\mathrm{ret} / \mathrm{adv}}\right)$, it can be absorbed by another mass counterterm

$$
\begin{aligned}
\delta m_{\mathrm{sun}}^{2} & =-\operatorname{Re} \Sigma_{T=0}^{c}\left(p^{2}\right)=-\operatorname{Re} \Sigma_{T=0}^{\mathrm{ret} / \mathrm{adv}}\left(p^{2}\right) \\
& =\frac{\lambda^{2}}{6} \int \frac{d^{2} q}{(2 \pi)^{2}} \int \frac{d^{2} r}{(2 \pi)^{2}} \frac{1}{4 \omega_{\boldsymbol{q}} \omega_{\boldsymbol{r}} \omega_{\boldsymbol{q}+\boldsymbol{r}-\boldsymbol{p}}} \frac{\omega_{\boldsymbol{q}}+\omega_{\boldsymbol{r}}+\omega_{\boldsymbol{q}+\boldsymbol{r}-\boldsymbol{p}}}{\left[\omega_{\boldsymbol{q}}+\omega_{\boldsymbol{r}}+\omega_{\boldsymbol{q}+\boldsymbol{r}-\boldsymbol{p}}\right]^{2}-p_{0}^{2}}
\end{aligned}
$$

at given 4-momentum $p=\left(p_{0}, \boldsymbol{p}\right)$ and renormalized mass $m$. 


\subsection{Numerical implementation}

For the solution of the Kadanoff-Baym equations we generate a closed set of first order differential equations in time for the Green's functions $i G_{\phi \phi}^{<}(x, y)=$ $\langle\phi(y) \phi(x)\rangle, i G_{\pi \phi}^{<}(x, y)=\langle\phi(y) \pi(x)\rangle$ and $i G_{\pi \pi}^{<}(x, y)=\langle\pi(y) \pi(x)\rangle$ with the canonical field momentum $\pi(x)=\partial_{x_{0}} \phi(x)$. The disadvantage of having more Green's functions in this scheme is compensated by its good accuracy. We especially take into account the propagation along the time diagonal which leads to an improved numerical precision. The set of differential equations is solved by means of a Runge-Kutta algorithm of 4th order. For the calculation of the self-energies we apply a Fourier method similar to the one used in [15,34]. The self-energies (17) are calculated in coordinate space, where they are products of coordinate-space Green's functions - that are available by Fourier transformation - and are finally transformed to momentum space again. We note that the momentum space is discretized by a finite number of momentum states fixed by periodic boundary conditions in a finite volume $V=a^{2}$.

\subsection{Equilibration}

As already observed in the $1+1$ dimensional case [29-31] the inclusion of the mean field does not lead to an equilibration of arbitrary initialized momentum distributions, since it only modifies the propagation of the particles by the generation of an effective mass. Thermalization in $2+1$ dimensions requires the inclusion of the collisional self-energies from the sunset diagram. To demonstrate the
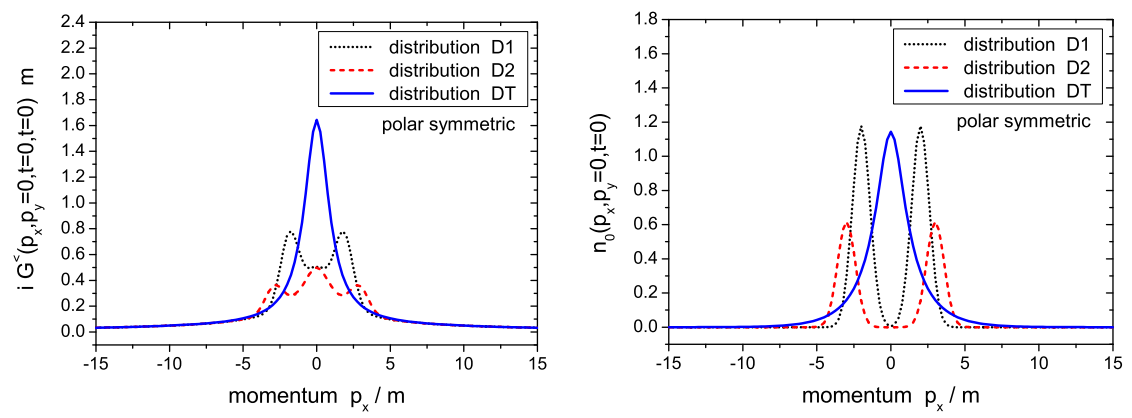

Fig. 2. Initial Green's functions $i G^{<}(|\boldsymbol{p}|, t=0, t=0)$ (left) and corresponding initial distribution functions $n_{o}(|\boldsymbol{p}|, t=0)$ (right) for the distributions D1, D2 and DT in momentum space (cut of the polar symmetric distribution in $p_{x}$ for $p_{y}=0$ )

phenomenon of equilibration we consider the time evolution of several initial conditions characterized by the same energy density. The initial equal-time Green's functions $i G^{<}(\boldsymbol{p}, t=0, t=0)$ adopted are displayed in Fig. 2 (l.h.s.) as a function of the momentum $p_{x}$ (for $p_{y}=0$ ). We concentrate on polar symmetric configurations due to the large numerical expense for this first investigation ${ }^{1}$. Since the

\footnotetext{
${ }^{1}$ In Section 3 we will present calculations for non-symmetric systems.
} 


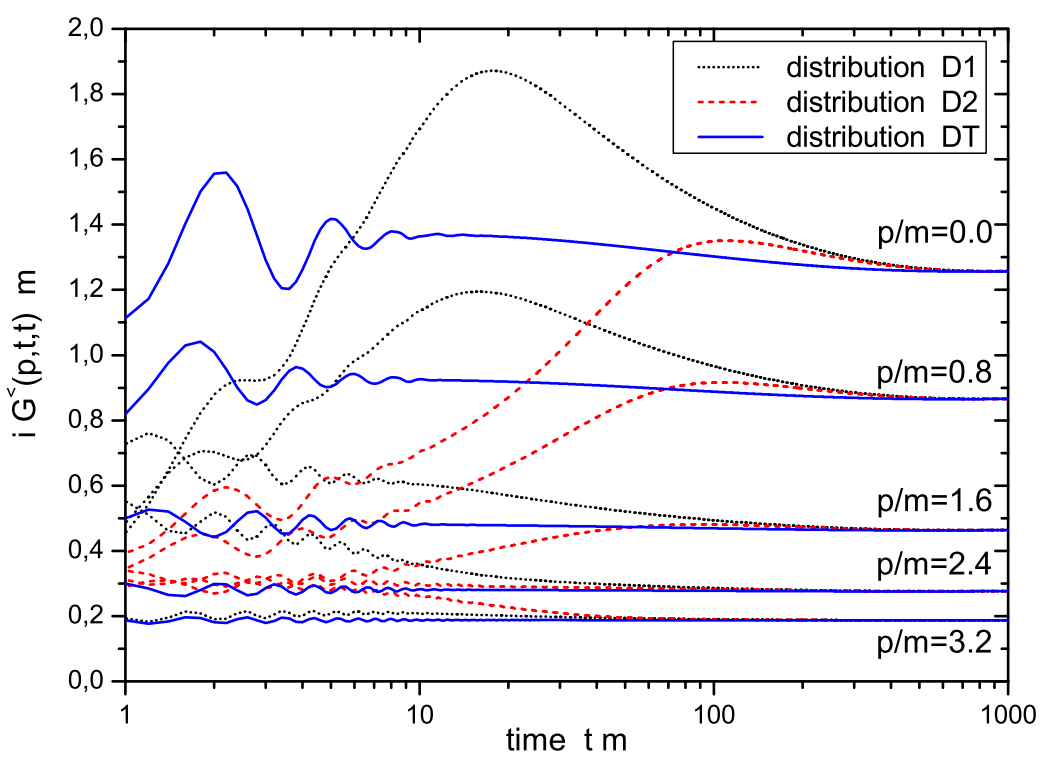

Fig. 3. Time evolution of various momentum modes of the equal-time Green's function for $|\boldsymbol{p}| / m=0.0,0.8,1.6,2.4,3.2$ (from top to bottom) for three different initial configurations D1, D2, DT (characterized by the different line type) with the same energy density. For the rather strong coupling constant $\lambda / m=16$ the initial oscillations are damped rapidly and have already disappeared at $t \cdot m=20$. Finally all momentum modes assume the same respective equilibrium value independent of the initial state

equal-time Green's functions $G^{<}(\boldsymbol{p}, t, t)$ are purely imaginary we display (the real part of) $i G^{<}$. Furthermore, the corresponding initial distribution functions $n_{o}(\boldsymbol{p}, t=0)$ are shown in Fig. 2 (r.h.s.). While the initial distributions D1, D2 have the shape of (polar symmetric) 'tsunami' waves with maxima at different momenta, the initial distribution DT corresponds to a free Bose gas at a given initial temperature. The difference between the Green's functions and the distribution functions is given by the vacuum contribution which has its maximum at small momenta, i.e. $2 \omega_{\boldsymbol{p}} i G_{\phi \phi}^{<}(\boldsymbol{p}, t=0, t=0)=2 n_{o}(\boldsymbol{p}, t=0)+1$. So even for the distributions D1, D2 the corresponding Green's functions are nonvanishing at low momentum. The time evolution of various momentum modes of the equaltime Green's function for these three different initial states is shown in Fig. 3. Starting from different initial conditions (as shown in Fig. 2) the single momentum modes finally approach the equilibrium values. For large times all modes reach a static limit as characteristic for an equilibrated system. On the other hand, for small times one observes a damped oscillating behaviour. This can be identified as a typical 'switching-on' effect where the system - being in a static (free) situation - is excited by a sudden increase of the coupling constant. In the present calculation the initial state is given by a free state with mass $m=1$ and 
the coupling $\lambda / m=16$ is switched on for times larger than 0 . One might also start with an effective initial mass due to the self-consistent tadpole contribution [31]. For the study of the equilibration process, however, this does not make any significant difference. Due to the character of the self-energies the 'switching-on' oscillations are damped in the course of time. The damping depends on the coupling strength and is much larger for strongly coupled systems. For very strong interactions the initial oscillations are even hard to recognize. The final part of the time evolution is characterized by an approximately exponential approach to the equilibrium value. In contrast to the calculations performed for $\phi^{4}$-theory in $1+1$ space-time dimensions [31] there is no intermediate region, where the momentum modes show a power-law behaviour. Furthermore, we observe that depending on the initial conditions and the coupling strength - the momentum modes can temporarily exceed their respective equilibrium value. This can be seen for the lowest momentum modes of distribution D1 in Fig. 3 which possesses initially a maximum at relatively small momentum. Especially the momentum mode $|\boldsymbol{p}|=0$ of the equal-time Green's function $G^{<}$, which starts at around 0.5 , is rising to a value of $\sim 1.8$ before it is decreasing again to its equilibrium value of about 1.26 . Thus the evolution towards the final equilibrium value is after the initial phase with damped oscillations - not necessarily monotonic. On the other hand, initial configurations like the distribution DT, where the system initially is given by a free gas of particles at a temperature $T_{0}$, do not show this property. Although they are - of course - not the equilibrium state of the interacting theory, they are much closer to it than the distributions D1 and D2. Therefore, the evolution towards the equilibrium distribution runs less violently.

\subsection{Time Evolution of the Spectral Function}

Within the Kadanoff-Baym calculations the full quantum character of the twopoint functions is taken into account. Consequently one fully incorporates the spectral properties of the nonequilibrium system during its time evolution. The spectral function $A(x, y)$ in our case is given by

$$
A(x, y)=\left\langle[\phi(x), \phi(y)]_{-}\right\rangle=i\left[G^{>}(x, y)-G^{<}(x, y)\right] .
$$

For each system time $T=\left(t_{1}+t_{2}\right) / 2$ the spectral function in Wigner-space is obtained via Fourier transformation with respect to the relative time coordinate $\Delta t=t_{1}-t_{2}$ :

$$
A\left(\boldsymbol{p}, p_{0}, T\right)=\int_{-\infty}^{\infty} d \Delta t e^{i \Delta t p_{0}} A\left(\boldsymbol{p}, t_{1}=T+\Delta t / 2, t_{2}=T-\Delta t / 2\right) .
$$

A damping of the function $A\left(\boldsymbol{p}, t_{1}, t_{2}\right)$ in relative time $\Delta t$ corresponds to the generation of a finite width of the spectral function in Wigner-space. This width in turn can be interpreted as the inverse life time of the scalar particle. We recall that the spectral function obeys - for every mean time $T$ and for all momenta $\boldsymbol{p}$ - the normalization

$$
\int_{-\infty}^{\infty} \frac{d p_{0}}{2 \pi} p_{0} A\left(\boldsymbol{p}, p_{0}, T\right)=1 \quad \forall \boldsymbol{p}, T
$$



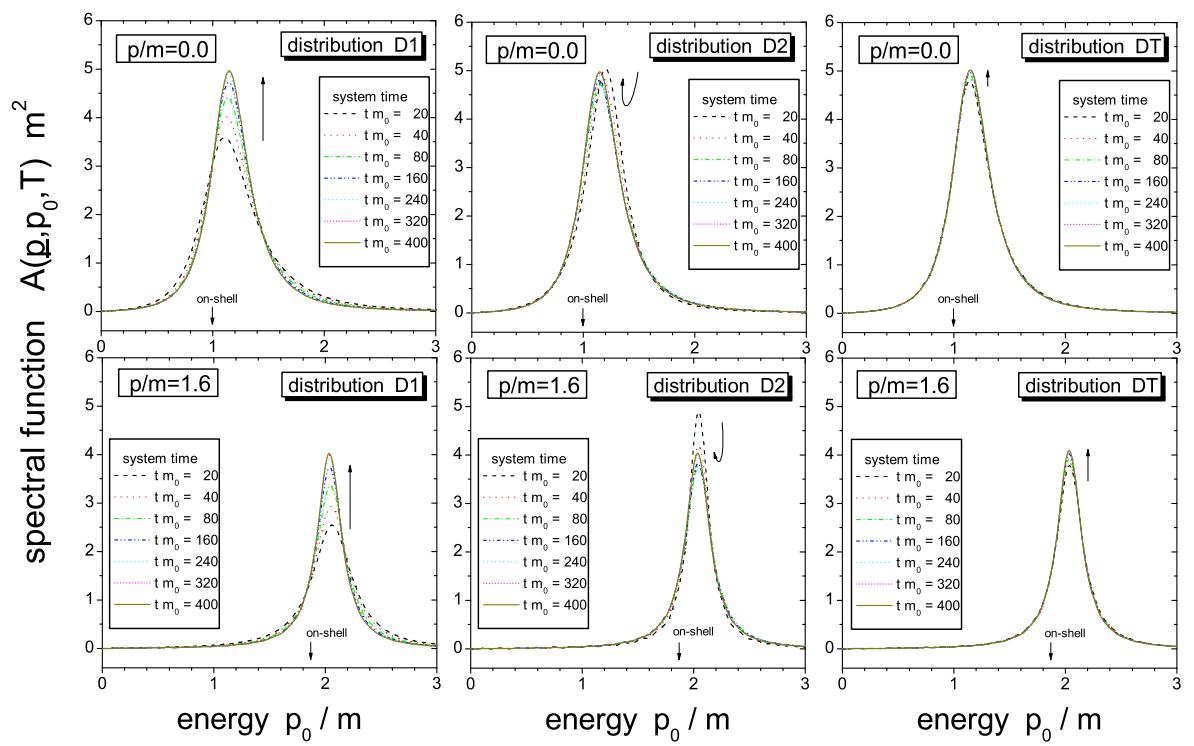

Fig. 4. Time evolution of the spectral function $A\left(\boldsymbol{p}, p_{0}, T\right)$ for the initial distributions D1, D2 and DT (from left to right) for the two momenta $|\boldsymbol{p}| / m=0.0$ (upper row) and $|\boldsymbol{p}| / m=1.6$ (lower row). The spectral function is shown for several times $t \cdot m=20$, 40, 80, 160, 240, 320, 400 as indicated by the different line types

which is a reformulation of the equal-time commutation relation (8).

In Fig. 4 we present the time evolution of the spectral function for the initial distributions D1, D2 and DT for two different momentum modes $|\boldsymbol{p}| / m=0.0$ and $|\boldsymbol{p}| / m=1$.6. Since the spectral functions are antisymmetric in energy $A\left(\boldsymbol{p},-p_{0}, T\right)=-A\left(\boldsymbol{p}, p_{0}, T\right)$ we only show the positive energy part. For our initial value problem in two-time space the Fourier transformation (21) is restricted for system time $T$ to an interval $\Delta t \in[-2 T, 2 T]$. Thus in the very early phase the spectral function assumes a finite width already due to the limited support of the Fourier transformation and a Wigner representation is not very meaningful. We therefore present the spectral functions for various system times starting from $t \cdot m=20$ up to $t \cdot m=400$.

For the free thermal initialization DT the evolution of the spectral function is very smooth. It is comparable to the smooth evolution of the equal-time Green's function for this initialization as discussed in the last Subsection. The spectral function is already close to the equilibrium shape at small times being initially only slightly broader than for late times. The maximum of the spectral function lies (for all momenta) higher than the (bare) on-shell value and nearly keeps its position over the whole time evolution. It results from a positive tadpole mass shift which is only partly compensated by a downward shift from the sunset diagram. 
The time evolution of the initial distributions D1 and D2 has a richer structure. For the initial distribution D1 the spectral function is broad for small system times (see the line for $t \cdot m=20$ ) and is getting a little sharper in the course of the system evolution (as presented for the momentum mode $|\boldsymbol{p}| / m=0.0$ as well as for $|\boldsymbol{p}| / m=1.6)$. At the same time the height of the spectral function is increasing (as demanded by the normalization property (22)) with time. This is indicated by the small arrow close to the peak position. Furthermore, the maximum of the spectral function (which is approximately the on-shell energy) is shifted slightly upwards for the zero mode and downwards for the mode with higher momentum. Although the real part of the (retarded) sunset self-energy leads (in general) to a lowering of the effective mass, the on-shell energy of the momentum modes is still higher than the one for initial mass $m$ (indicated by the 'on-shell' arrow) due to the positive mass shift from the tadpole contribution. For the initial distribution D2 we find an opposite behaviour. Here the spectral function develops at intermediate times a slightly higher width than in the beginning before it is approaching the narrower static shape. The corresponding evolution of the maximum is again indicated by the (bent) arrow. Finally all spectral functions assume the (same) equilibrium form.

As already observed above for the equal-time Green's functions, we emphazise that there is no unique type of evolution for the nonequilibrium system. In fact, the evolution of the system during the equilibration process is sensitive to the initial conditions.

\subsection{Generation of Correlations}

The time evolution within the Kadanoff-Baym equation is characterized by the generation of correlations. This can be seen from Fig. 5, where all energy density contributions are displayed as a function of time. The kinetic energy density $\varepsilon_{\text {kin }}$ is given by all parts of the total energy density that do not depend on the coupling constant $\left(\propto \lambda^{0}\right)$. All terms proportional to $\lambda^{1}$ are summarized by the tadpole energy density $\varepsilon_{\text {tad }}$ including the actual tadpole term as well as the corresponding tadpole mass counterterm. The contributions from the sunset diagram $\left(\propto \lambda^{2}\right)$ - given by the correlation integral $I_{1}^{<}$as well as by the sunset mass counterterm - are represented by the sunset energy density $\varepsilon_{\text {sun }},{ }^{2}$

$$
\begin{aligned}
& \varepsilon_{\text {tot }}(t)=\varepsilon_{\text {kin }}(t)+\varepsilon_{\text {tad }}(t)+\varepsilon_{\text {sun }}(t), \\
& \varepsilon_{\text {kin }}(t)=\frac{1}{2} \int \frac{d^{d} p}{(2 \pi)^{d}}\left(\boldsymbol{p}^{2}+m^{2}\right) i G_{\phi \phi}^{<}(\boldsymbol{p}, t, t)+\frac{1}{2} \int \frac{d^{d} p}{(2 \pi)^{d}} i G_{\pi \pi}^{<}(\boldsymbol{p}, t, t), \\
& \varepsilon_{\mathrm{tad}}(t)=\frac{1}{4} \int \frac{d^{d} p}{(2 \pi)^{d}} \bar{\Sigma}_{\mathrm{tad}}(t) i G_{\phi \phi}^{<}(\boldsymbol{p}, t, t)+\frac{1}{2} \int \frac{d^{d} p}{(2 \pi)^{d}} \delta m_{\mathrm{tad}}^{2} i G_{\phi \phi}^{<}(\boldsymbol{p}, t, t),
\end{aligned}
$$

${ }^{2}$ Speaking of powers of the coupling constant we mean the 'superficial' order of the corresponding diagrams. Since our self-energies $\Sigma$ are built up self-consistently by full Green's functions $G$ always higher orders of the coupling constant are resummed. 


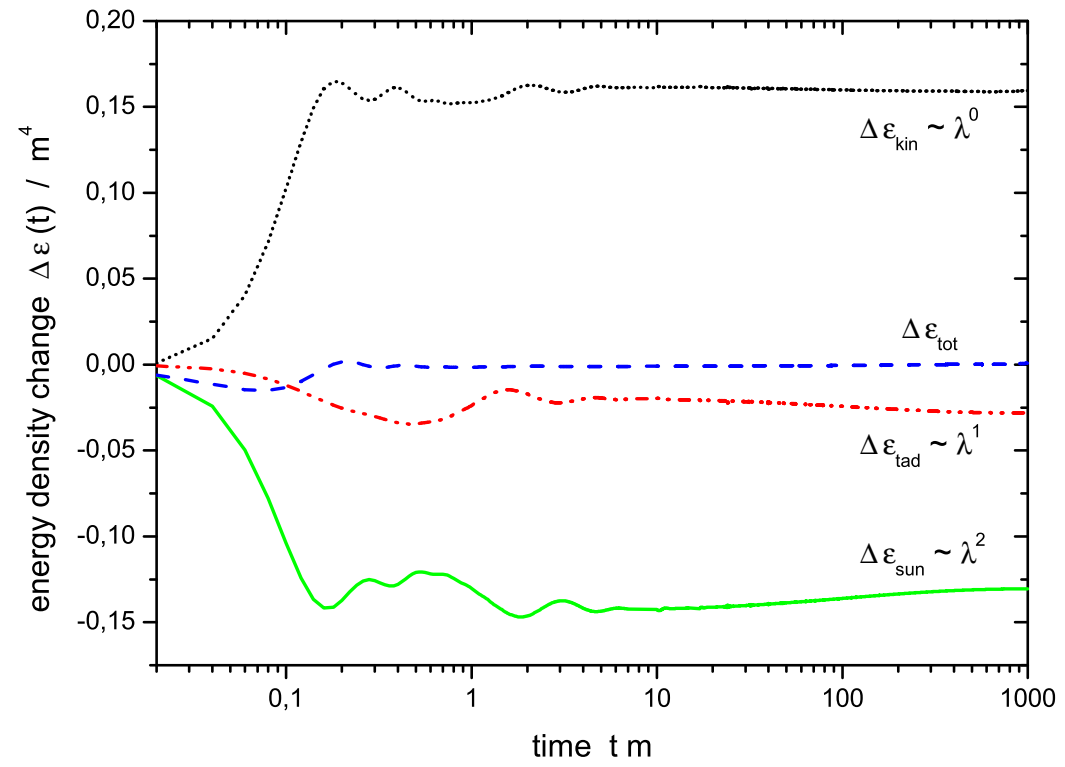

Fig. 5. Change of the different contributions to the total energy density in time. The sunset energy density $\varepsilon_{\text {sun }}$ decreases as the system correlates. This is mostly compensated by an increase of the kinetic energy density $\varepsilon_{\text {kin }}$. Together with the smaller tadpole contribution $\varepsilon_{\text {tad }}$ the total energy $\varepsilon_{\text {tot }}$ is conserved

$\varepsilon_{\mathrm{sun}}(t)=-\frac{1}{4} \int \frac{d^{d} p}{(2 \pi)^{d}} i I_{1}^{<}(\boldsymbol{p}, t, t)+\frac{1}{2} \int \frac{d^{d} p}{(2 \pi)^{d}} \delta m_{\mathrm{sun}}^{2} i G_{\phi \phi}^{<}(\boldsymbol{p}, t, t)$.

The calculation in Fig. 5 has been performed for the initial distribution DT (which corresponds to a free gas of Bose particles at temperature $T_{0}=1.59 \mathrm{~m}$ ) with a coupling constant $\lambda / m=16$. This state is a stationary solution for the well-known Boltzmann equation, but not for the Kadanoff-Baym equation. In the latter treatment the system evolves from the uncorrelated state and the correlation energy density $\varepsilon_{\text {sun }}$ decreases with time. The decrease of the correlation energy $\varepsilon_{\text {sun }}$, which is - with exception of the sunset mass counterterm contribution - initially zero, is mainly compensated by an increase of the kinetic energy density $\varepsilon_{\text {kin }}$. The remaining difference is equal to the change of the tadpole energy density $\varepsilon_{\text {tad }}$ such that the total energy density is conserved. While the sunset and the kinetic term have always the behaviour displayed in the figure, the change of the tadpole energy density depends on the initial configuration and can be positive or negative. Since the self-energies are obtained within a $\Phi$ derivable scheme (9), the fundamental conservation laws, as e.g. energy conservation, are respected to all orders in the coupling constant [32]. 


\section{Comparison to the Boltzmann Limit}

As mentioned above repeatedly the Kadanoff-Baym equation represents the full quantum field theoretical dynamics on the one-particle level. However, its numerical solution is quite involved and it is of strong interest to investigate, in how far approximate schemes deviate from the full calculation. Nowadays, transport models are widely used in the description of quantum system out of equilibrium (cf. Introduction). Most of these models work in the 'quasiparticle' picture, where all particles obey a fixed energy-momentum relation and the energy is no independent degree of freedom anymore; it is determined by the momentum and the (effective) mass of the particle. Accordingly, these particles are treated with their $\delta$-function spectral shape as infinitely long living, i.e. stable objects. This assumption is rather questionable e.g. for high-energy heavy ion reactions, where the particles achieve a large width due to the frequent collisions with other particles in the high density and/or high energy regime. Furthermore, this is doubtful for particles that are unstable even in the vacuum. The question, in how far the quasiparticle approximation influences the dynamics in comparison to the full Kadanoff-Baym calculation, is of general interest [15,34].

In order to investigate this question we formulate the Boltzmann limit in analogy to Ref. [35]. For the detailed steps and assumptions in the actual derivation we refer the reader to Ref. [36]. The Boltzmann equation describes the time evolution of the momentum distribution function

$$
N(\boldsymbol{p}, t)=\frac{\omega_{\boldsymbol{p}}}{2} i G_{\phi \phi}^{<}(\boldsymbol{p}, t, t)+\frac{1}{2 \omega_{\boldsymbol{p}}} i G_{\pi \pi}^{<}(\boldsymbol{p}, t, t)-\operatorname{Re} G_{\pi \phi}^{<}(\boldsymbol{p}, t, t)
$$

by $2 \leftrightarrow 2$ on-shell scattering processes, i.e.

$$
\begin{aligned}
\partial_{t} N(\boldsymbol{p}, t)= & \frac{\lambda^{2}}{2 \omega_{\boldsymbol{p}}} \int \frac{d^{d} q}{(2 \pi)^{d}} \int \frac{d^{d} r}{(2 \pi)^{d}} \int \frac{d^{d} s}{(2 \pi)^{d}} \frac{1}{8 \omega_{\boldsymbol{q}} \omega_{\boldsymbol{r}} \omega_{\boldsymbol{s}}}(2 \pi)^{d} \delta^{(d)}(\boldsymbol{p}+\boldsymbol{q}-\boldsymbol{r}-\boldsymbol{s}) \\
& \left\{\bar{N}_{\boldsymbol{p}} \bar{N}_{\boldsymbol{q}} N_{\boldsymbol{r}} N_{\boldsymbol{s}}-N_{\boldsymbol{p}} N_{\boldsymbol{q}} \bar{N}_{\boldsymbol{r}} \bar{N}_{\boldsymbol{s}}\right\} \pi \delta\left(\omega_{\boldsymbol{p}}+\omega_{\boldsymbol{q}}-\omega_{\boldsymbol{r}}-\omega_{\boldsymbol{s}}\right)
\end{aligned}
$$

using $N_{\boldsymbol{p}}=N(\boldsymbol{p}, t)$ and $\bar{N}_{\boldsymbol{p}}=N(\boldsymbol{p}, t)+1$ for the corresponding Bose factors.

The numerical procedure for the solution of (25) is basically the same as the one developed for the solution of the Kadanoff-Baym equation. Moreover, we calculate the actual momentum dependent on-shell energy $\omega_{\boldsymbol{p}}$ for every momentum mode by a solution of the dispersion relation including contributions from the tadpole and the real part of the (retarded) sunset self-energy. Thus it is guaranteed, that the particles are treated as quasiparticles with the correct energy-momentum relation at every time.

For the comparison between the full Kadanoff-Baym dynamics and the Boltzmann approximation we concentrate on equilibration times. As a measure for the equilibration time we consider the time scale on which the initially nonisotropic distribution proceeds to the polar symmetric equilibrium value. We 


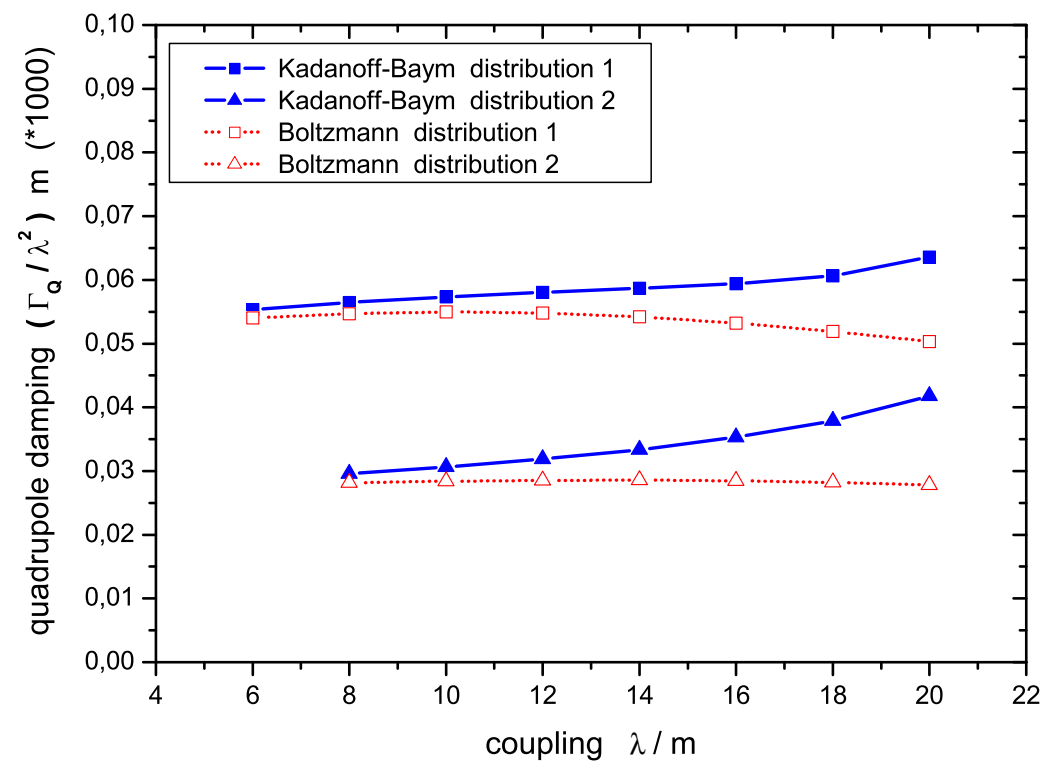

Fig. 6. Relaxation rate (divided by the coupling squared) for Kadanoff-Baym and Boltzmann calculations as a function of the interaction strength. For the two different initial configurations the full Kadanoff-Baym evolution leads to a faster equilibration

define a moment $Q(t)$ for a given momentum distribution $n(\boldsymbol{p}, t)$ at time $t$ by

$$
Q(t)=\frac{\int \frac{d^{2} p}{(2 \pi)^{2}}\left(p_{x}^{2}-p_{y}^{2}\right) n(\boldsymbol{p}, t)}{\int \frac{d^{2} p}{(2 \pi)^{2}} n(\boldsymbol{p}, t)},
$$

which vanishes for symmetric systems, e.g. for the equilibrium state. For the Kadanoff-Baym case we calculate the actual distribution function by

$$
n(\boldsymbol{p}, t)=\sqrt{G_{\phi \phi}^{<}(\boldsymbol{p}, t, t) G_{\pi \pi}^{<}(\boldsymbol{p}, t, t)}-\frac{1}{2} .
$$

The moment $Q(t)$ shows an approximately exponential decrease in time such that we can define a relaxation rate $\Gamma_{Q}$ via the relation $Q(T) \propto e^{-\Gamma_{Q} t}$. In Fig. 6 the relaxation rate $\Gamma_{Q}$ (scaled by the coupling strength squared) is displayed for the Kadanoff-Baym and the Boltzmann calculation for two different initial configurations. The initial distribution 2 corresponds to the initial state of two (on the $p_{x}$-axis) separated particle accummulations (cf. Fig. 7). The peak at low momenta again comes from the vacuum contribution of the Green's function. The time evolution within the full Kadanoff-Baym theory is presented in Fig. 7 by several snapshots at times $t \cdot m=0,10.5,21,35,70,105$ showing the propagation towards an isotropic (static) final state. For distribution 1 the position and the width of the two bumps is modified. 

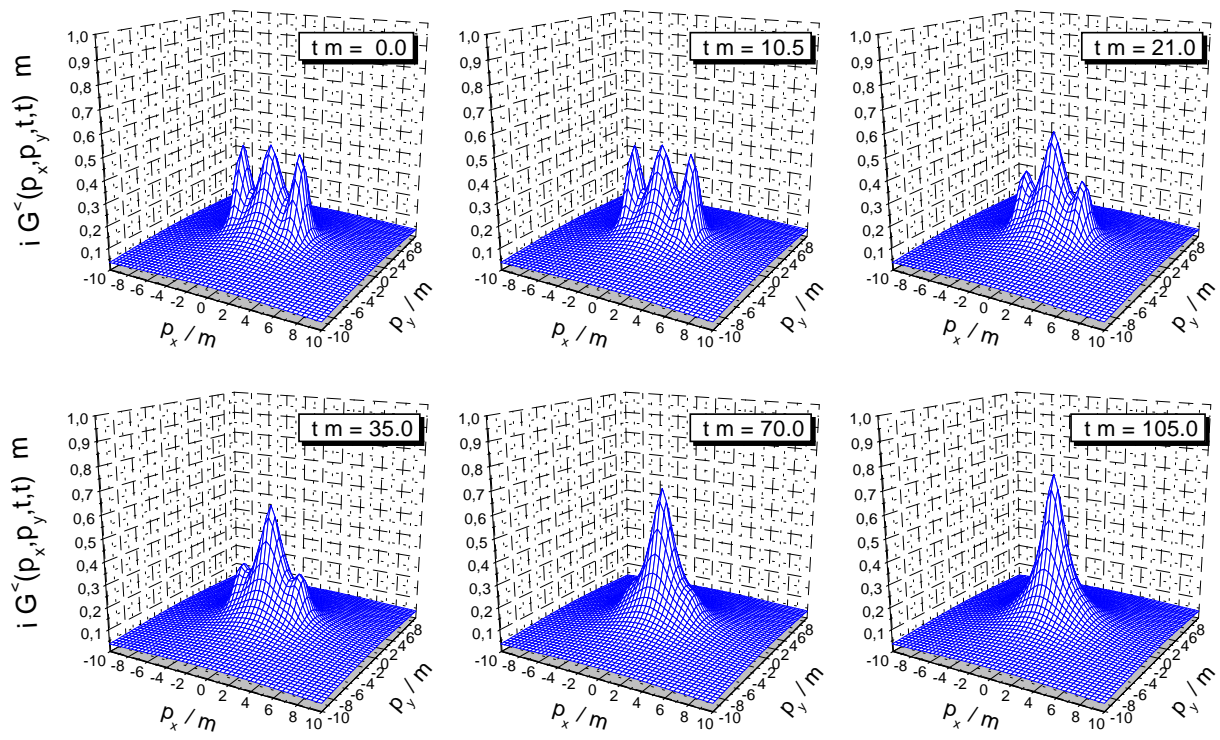

Fig. 7. Evolution of the Green's function in momentum space. The equal time Green's function is displayed for various times $t \cdot m=0,10.5,21,35,70,105$. Starting from an initially non-isotropic shape it develops towards a symmetric final distribution

Fig. 6 shows for both initializations that the relaxation in the full quantum calculation happens faster for large coupling constants than in the quasi-classical approximation, whereas for small couplings the equilibration times of the full and the approximate evolutions are comparable. While the scaled relaxation rate $\Gamma_{Q} / \lambda^{2}$ is nearly constant in the Boltzmann case, it increases with the coupling strength in the Kadanoff-Baym calculation (especially for initial distribution 2).

Since the free Green's function - as used in the Boltzmann calculation - has only support on the mass shell, only $(2 \leftrightarrow 2)$ scattering processes are described in the Boltzmann limit. All other processes with a different number of incoming and outgoing particles vanish. Within the full Kadanoff-Baym calculation this is very much different since here the spectral function - determined from the self-consistent Green's function - aquires a finite width. The Green's function has support at all energies - although it drops far off the mass shell. Especially for large coupling constants, where the spectral function is sufficiently broad, the three particle production process gives a significant contribution to the collision integral. Since the width of the spectral function increases with the interaction strength, such processes become more important in the high coupling regime. As a consequence the difference between both approaches is larger for stronger interactions as observed in Fig. 6. For small couplings $\lambda / m$ in both approaches 
basically the usual $2 \leftrightarrow 2$ scattering contributes and the results for the thermalization rate $\Gamma_{Q}$ are quite similar.

In summarizing this Section we point out that the full solution of the KadanoffBaym equations does include $0 \leftrightarrow 4,1 \leftrightarrow 3$ and $2 \leftrightarrow 2$ off-shell collision processes which - in comparison to the Bolzmann on-shell $2 \leftrightarrow 2$ collision limit - become important when the spectral width of the particles reaches about $1 / 3$ of the particle mass. On the other hand, the simple Boltzmann limit works surprisingly well for smaller couplings and those cases, where the spectral function is sufficiently narrow.

\section{Derivation of Semiclassical Transport Equations for Particles with Dynamical Life Times}

The Kadanoff-Baym equations (5) presently cannot be solved for general inhomogeneous problems due to the high complexity of these equations and the huge 'computer storage' required. It is thus of interest to obtain a semiclassical limit which is much easier to solve e.g. by current testparticle methods.

We start again with the Kadanoff-Baym equation (5), however, change to the Wigner representation via Fourier transformation of the rapidly oscillating relative coordinate $(x-y)$. The theory is then formulated in terms of the coordinates $X=(x+y) / 2$ and the momentum $p$,

$$
F_{X p}=\int d^{4}(x-y) e^{i p_{\mu}\left(x^{\mu}-y^{\mu}\right)} F_{x y} .
$$

Since convolution integrals convert under Wigner transformations as

$$
\int d^{4}(x-y) e^{i p_{\mu}\left(x^{\mu}-y^{\mu}\right)} F_{1, x z} \odot F_{2, z y}=e^{-i \diamond} F_{1, X p} F_{2, X p},
$$

one has to deal with an infinite series in the differential operator $\diamond$ which is a four-dimensional generalization of the Poisson-bracket,

$$
\diamond\left\{F_{1}\right\}\left\{F_{2}\right\}=\frac{1}{2}\left(\frac{\partial F_{1}}{\partial X_{\mu}} \frac{\partial F_{2}}{\partial p^{\mu}}-\frac{\partial F_{1}}{\partial p_{\mu}} \frac{\partial F_{2}}{\partial X^{\mu}}\right) .
$$

As a standard approximation of kinetic theory only contributions up to first order in the gradients gradient expansion are considered. This is justified if the gradients in the mean spatial coordinate $X$ and momentum $p$ are small. Applying this approximation scheme (Wigner transformation and neglecting all gradient terms of order $n \geq 2$ ) to the Dyson-Schwinger equations of the retarded and advanced Green's functions one ends up with

$$
\begin{aligned}
&\left(p^{2}-M_{0}^{2}-\operatorname{Re} \Sigma_{X p}^{\mathrm{ret}}\right) \operatorname{Re} G_{X p}^{\mathrm{ret}}=1-\frac{1}{4} \Gamma_{X p} A_{X p}, \\
&\left(p^{2}-M_{0}^{2}-\operatorname{Re} \Sigma_{X p}^{\mathrm{ret}}\right) A_{X p}=\Gamma_{X p} \operatorname{Re} G_{X p}^{\mathrm{ret}},
\end{aligned}
$$


where we have separated the retarded and advanced Green's functions as well as the self-energies into real and imaginary contributions

$$
G_{X p}^{\mathrm{ret}, \mathrm{adv}}=\operatorname{Re} G_{X p}^{\mathrm{ret}} \mp \frac{i}{2} A_{X p}, \quad \Sigma_{X p}^{\mathrm{ret}, \mathrm{adv}}=\operatorname{Re} \Sigma_{X p}^{\mathrm{ret}} \mp \frac{i}{2} \Gamma_{X p} .
$$

The imaginary part of the retarded propagator is given (up to a factor) by the normalized spectral function $A_{X p}(20)$ while the (negative) imaginary part of the self-energy is half the width $\Gamma_{X p}$. Above equations (31) are given for a (renormalized) vacuum mass $M_{0}$ and a (renormalized) retarded self-energy $\Sigma^{\text {ret }}$. In the presence of an additional space-time local self-energy (e.g. tadpole self-energy) the mass term is shifted accordingly $M_{0}^{2} \rightarrow M_{0}^{2}+\Sigma_{\text {tad. }}$. From the algebraic equations (31) we obtain a direct relation between the real and the imaginary part of the propagator (provided $\Gamma_{X p} \neq 0$ ):

$$
\operatorname{Re} G_{X p}^{\mathrm{ret}}=\frac{p^{2}-M_{0}^{2}-\operatorname{Re} \Sigma_{X p}^{\mathrm{ret}}}{\Gamma_{X p}} A_{X p}
$$

The algebraic solution for the spectral function shows a Lorentzian shape with space-time and four-momentum dependent width $\Gamma_{X p}$. This result is valid for bosons to first order in the gradient expansion,

$$
A_{X p}=\frac{\Gamma_{X p}}{\left(p^{2}-M_{0}^{2}-\operatorname{Re} \Sigma_{X p}^{\mathrm{ret}}\right)^{2}+\Gamma_{X p}^{2} / 4} .
$$

For the real part of the retarded Green's function we get also algebraically

$$
\operatorname{Re} G_{X p}^{\mathrm{ret}}=\frac{p^{2}-M_{0}^{2}-\operatorname{Re} \Sigma_{X p}^{\mathrm{ret}}}{\left(p^{2}-M_{0}^{2}-\operatorname{Re} \Sigma_{X p}^{\mathrm{ret}}\right)^{2}+\Gamma_{X p}^{2} / 4} .
$$

\subsection{Transport Equations}

The Kadanoff-Baym equation (5) gives in the same semiclassical approximation scheme a generalized transport equation,

$$
\begin{gathered}
\diamond\left\{p^{2}-M_{0}^{2}-\operatorname{Re} \Sigma_{X p}^{\mathrm{ret}}\right\}\left\{G_{X p}^{<}\right\}-\diamond\left\{\Sigma_{X p}^{<}\right\}\left\{\operatorname{Re} G_{X p}^{\mathrm{ret}}\right\} \\
=\frac{i}{2}\left[\Sigma_{X p}^{>} G_{X p}^{<}-\Sigma_{X p}^{<} G_{X p}^{>}\right]
\end{gathered}
$$

and a generalized mass-shell equation,

$$
\begin{aligned}
& {\left[p^{2}-M_{0}^{2}-\operatorname{Re} \Sigma_{X p}^{\mathrm{ret}}\right] G_{X p}^{<}-\Sigma_{X p}^{<} \operatorname{Re} G_{X p}^{\mathrm{ret}}} \\
& \quad=\frac{1}{2} \diamond\left\{\Sigma_{X p}^{<}\right\}\left\{A_{X p}\right\}-\frac{1}{2} \diamond\left\{\Gamma_{X p}\right\}\left\{G_{X p}^{<}\right\} .
\end{aligned}
$$

In the transport equation (36) one recognizes on the l.h.s. the drift term $p^{\mu} \partial_{\mu} \bullet$ as generated by the contribution $\diamond\left\{p^{2}-M_{0}^{2}\right\}\{\bullet\}$, as well as the Vlasov term determined by the real part of the retarded self-energy. On the other hand the 
r.h.s. represents the collision term with its 'gain and loss' structure. To evaluate the $\diamond\left\{\Sigma^{<}\right\}\left\{\operatorname{Re} G^{\text {ret }}\right\}$-term in (36), which does not contribute in the quasiparticle limit, it is useful to introduce distribution functions for the Green's functions and self-energies as

$$
\begin{array}{ll}
i G_{X p}^{<}=N_{X p} A_{X p}, & i G_{X p}^{>}=\left(1+N_{X p}\right) A_{X p}, \\
i \Sigma_{X p}^{<}=N_{X p}^{\Sigma} \Gamma_{X p}, & i \Sigma_{X p}^{>}=\left(1+N_{X p}^{\Sigma}\right) \Gamma_{X p} .
\end{array}
$$

Following the argumentation of Botermans and Malfliet [16] the distribution functions $N$ and $N^{\Sigma}$ in (38) should be equal in the second term of the l.h.s. of (36) within a consistent first order gradient expansion. As a consequence the self-energy $\Sigma^{<}$can be replaced by $G^{<} \cdot \Gamma / A$ in the term $\diamond\left\{\Sigma^{<}\right\}\left\{\operatorname{Re} G^{\text {ret }}\right\}$. The general transport equation (36) then can be written as

$$
\begin{aligned}
& {\left[\diamond\left\{p^{2}-M_{0}^{2}-\operatorname{Re} \Sigma_{X p}^{\mathrm{ret}}\right\}\left\{G_{X p}^{<}\right\}-\frac{1}{\Gamma_{X p}} \diamond\left\{\Gamma_{X p}\right\}\left\{\left(p^{2}-M_{0}^{2}-\operatorname{Re} \Sigma_{X p}^{\mathrm{ret}}\right) G_{X p}^{<}\right\}\right]} \\
& \times A_{X p} \Gamma_{X p}=i\left[\Sigma_{X p}^{>} G_{X p}^{<}-\Sigma_{X p}^{<} G_{X p}^{>}\right] .
\end{aligned}
$$

\subsection{Test Particle Representation}

In order to obtain an approximate solution to the transport equation (39) we use a test particle ansatz for the Green's function $G^{<}$, more specifically for the real and positive semidefinite quantity

$$
F_{X p}=A_{X p} N_{X p}=i G_{X p}^{<} \sim \sum_{i=1}^{N} \delta^{(3)}\left(\boldsymbol{X}-\boldsymbol{X}_{i}(t)\right) \delta^{(3)}\left(\boldsymbol{p}-\boldsymbol{p}_{i}(t)\right) \delta\left(p_{0}-\epsilon_{i}(t)\right) .
$$

In the most general case (where the self-energies depend on four-momentum $p$, time $t$ and the spatial coordinates $\boldsymbol{X}$ ) the equations of motion for the test particles read

$$
\begin{aligned}
& \frac{d \boldsymbol{X}_{i}}{d t}=\frac{1}{1-C_{(i)}} \frac{1}{2 \epsilon_{i}}\left[2 \boldsymbol{p}_{i}+\nabla_{p_{i}} \operatorname{Re} \Sigma_{(i)}^{\mathrm{ret}}+\frac{\epsilon_{i}^{2}-\boldsymbol{p}_{i}^{2}-M_{0}^{2}-\operatorname{Re} \Sigma_{(i)}^{\mathrm{ret}}}{\Gamma_{(i)}} \nabla_{p_{i}} \Gamma_{(i)}\right], \\
& \frac{d \boldsymbol{p}_{i}}{d t}=\frac{-1}{1-C_{(i)}} \frac{1}{2 \epsilon_{i}}\left[\boldsymbol{\nabla}_{X_{i}} \operatorname{Re} \Sigma_{i}^{\mathrm{ret}}+\frac{\epsilon_{i}^{2}-\boldsymbol{p}_{i}^{2}-M_{0}^{2}-\operatorname{Re} \Sigma_{(i)}^{\mathrm{ret}}}{\Gamma_{(i)}} \nabla_{X_{i}} \Gamma_{(i)}\right], \\
& \frac{d \epsilon_{i}}{d t}=\frac{1}{1-C_{(i)}} \frac{1}{2 \epsilon_{i}}\left[\frac{\partial \operatorname{Re} \Sigma_{(i)}^{\mathrm{ret}}}{\partial t}+\frac{\left.\epsilon_{i}^{2}-\boldsymbol{p}_{i}^{2}-M_{0}^{2}-\operatorname{Re} \Sigma_{(i)}^{\mathrm{ret}} \frac{\partial \Gamma_{(i)}}{\partial t}\right],}{\Gamma_{(i)}}\right.
\end{aligned}
$$

where the notation $F_{(i)}$ implies that the function is taken at the coordinates of the test particle at time $t$, i.e. $F_{(i)} \equiv F\left(t, \boldsymbol{X}_{i}(t), \boldsymbol{p}_{i}(t), \epsilon_{i}(t)\right)$. 
In (41-43) a common multiplication factor $\left(1-C_{(i)}\right)^{-1}$ appears, which contains the energy derivatives of the retarded self-energy

$$
C_{(i)}=\frac{1}{2 \epsilon_{i}}\left[\frac{\partial}{\partial \epsilon_{i}} \operatorname{Re} \Sigma_{(i)}^{\mathrm{ret}}+\frac{\epsilon_{i}^{2}-\boldsymbol{p}_{i}^{2}-M_{0}^{2}-\operatorname{Re} \Sigma_{(i)}^{\mathrm{ret}}}{\Gamma_{(i)}} \frac{\partial}{\partial \epsilon_{i}} \Gamma_{(i)}\right] .
$$

It yields a shift of the system time $t$ to the 'eigentime' of particle $i$ defined by $\tilde{t}_{i}=t /\left(1-C_{(i)}\right)$. As the reader immediately verifies, the derivatives with respect to the 'eigentime', i.e. $d \boldsymbol{X}_{i} / d \tilde{t}_{i}, d \boldsymbol{p}_{i} / d \tilde{t}_{i}$ and $d \epsilon_{i} / d \tilde{t}_{i}$ then emerge without this renormalization factor for each test particle $i$ when neglecting higher order time derivatives in line with the semiclassical approximation scheme. In the limiting case of particles with vanishing gradients of the width $\Gamma_{X p}$ these equations of motion reduce to the well-known transport equations of the quasiparticle picture.

Following Refs. [37] we take $M^{2}=p^{2}-\operatorname{Re} \Sigma^{\text {ret }}$ as an independent variable instead of $p_{0}$, which then fixes the energy (for given $\boldsymbol{p}$ and $M^{2}$ ) to

$$
p_{0}^{2}=\boldsymbol{p}^{2}+M^{2}+\operatorname{Re} \Sigma_{X \boldsymbol{p} M^{2}}^{\mathrm{ret}} .
$$

Eq. (43) then turns to

$$
\frac{d\left(M_{i}^{2}-M_{0}^{2}\right)}{d t}=\frac{M_{i}^{2}-M_{0}^{2}}{\Gamma_{(i)}} \frac{d \Gamma_{(i)}}{d t}
$$

for the time evolution of the test particle $i$ in the invariant mass squared as derived in Refs. [37]. For applications of the semiclassical off-shell transport approach we refer the reader to Refs. [37].

\section{Summary}

In this article we have studied the time evolution of an interacting field theoretical system, i.e. $\phi^{4}$-field theory in $2+1$ space-time dimensions, on the basis of the Kadanoff-Baym equations for a spatially homogeneous system including the self-consistent tadpole and sunset self-energies. We find that equilibration is achieved only by inclusion of the sunset self-energy. Simultaneously, the time evolution of the single-particle spectral function has been calculated for various initial conditions. A comparison of the full solution of the Kadanoff-Baym equations with the solution for the corresponding Boltzmann equation shows that a consistent inclusion of the spectral function has a sizeable impact on the equilibration rates if the width of the spectral function becomes larger than $\sim 1 / 3$ of the particle mass.

Furthermore, the conventional transport of particles in the on-shell quasiparticle limit has been extended to particles of finite life time by means of a dynamical spectral function $A\left(X, \boldsymbol{p}, M^{2}\right)$. Starting again from the Kadanoff-Baym equations we have derived in consistent first order gradient expansion equations of motion for test particles with respect to their time evolution in $\boldsymbol{X}, \boldsymbol{p}$ and $M^{2}$. This off-shell propagation has been examined for a couple of model cases in 
Refs. [37] as well as for nucleus-nucleus collisions showing that - at subthreshold energies - the off-shell dynamics play an important role for the production of energetic particles.

\section{Acknowledgements}

The authors acknowledge valuable discussions with C. Greiner and S. Leupold throughout these studies.

\section{References}

1. J. Schwinger, J. Math. Phys. 2, 407 (1961).

2. S. J. Wang and W. Cassing, Ann. Phys. (N.Y.) 159, 328 (1985).

3. W. Cassing and S. J. Wang, Z. Phys. A 337, 1 (1990).

4. K. Chou, Z. Su, B. Hao, and L. Yu, Phys. Rept. 118, 1 (1985).

5. S. Mrówczyński and P. Danielewicz, Nucl. Phys. B 342, 345 (1990).

6. S. Mrówczyński and U. Heinz, Ann. Phys. (N.Y.) 229, 1 (1994).

7. H. Stöcker and W. Greiner, Phys. Rept. 137, 277 (1986).

8. G. F. Bertsch and S. Das Gupta, Phys. Rept. 160, 189 (1988).

9. W. Cassing, V. Metag, U. Mosel, and K. Niita, Phys. Rept. 188, 363 (1990).

10. W. Cassing and U. Mosel, Prog. Part. Nucl. Phys. 25, 235 (1990).

11. C. Fuchs and T. Gaitanos, nucl-th/0211091; this volume.

12. S. Bass, M. Belkacem, M. Bleicher et al., Prog. Part. Nucl. Phys. 41, 255 (1998).

13. W. Cassing and E. L. Bratkovskaya, Phys. Rept. 308, 65 (1999).

14. L. P. Kadanoff and G. Baym, Quantum statistical mechanics, Benjamin, New York, 1962.

15. P. Danielewicz, Ann. Phys. (N.Y.) 152, 239 (1984); ibid. 305.

16. W. Botermans and R. Malfliet, Phys. Rept. 198, 115 (1990).

17. R. Malfliet, Prog. Part. Nucl. Phys. 21, 207 (1988).

18. P. A. Henning, Nucl. Phys. A 582, 633 (1995); Phys. Rept. 253, 235 (1995); this volume.

19. C. Greiner and S. Leupold, Ann. Phys. (N.Y.) 270, 328 (1998).

20. S. J. Wang, W. Zuo, and W. Cassing, Nucl. Phys. A 573, 245 (1994).

21. W. Cassing, K. Niita, and S. J. Wang, Z. Phys. A 331, 439 (1988).

22. R. Malfliet, Nucl. Phys. A 545, 3 (1992).

23. R. Malfliet, Phys. Rev. B 57, R11027 (1998).

24. P. Danielewicz and S. Pratt, Phys. Rev. C 53, 249 (1996).

25. V. Špička, P. Lipavský, and K. Morawetz, Phys. Rev. B 55, 5095 (1997); Phys. Lett. A 240, 160 (1998); this volume.

26. P. Lipavský, V. Špička, and K. Morawetz, Phys. Rev. E 59, 1291 (1999).

27. P. Lipavský, K. Morawetz, and V. Špička, Annales de Physique 26, 1 (2001).

28. A. Peter et al., Z. Phys. A 358, 91 (1997); Z. Phys. C 71, 515 (1997).

29. J. Berges and J. Cox, Phys. Lett. B 517, 369 (2001).

30. J. Berges and G. Aarts, Phys. Rev. D 64, 105010 (2001).

31. J. Berges, Nucl. Phys. A 699, 847 (2002).

32. Y. B. Ivanov, J. Knoll, and D. N. Voskresensky, Nucl. Phys. A 657, 413 (1999).

33. H. van Hees and J. Knoll, Phys. Rev. D 65, 025010 (2002); Phys. Rev. D 65, 105005 (2002).

34. H. S. Köhler, Phys. Rev. C 51, 3232 (1995).

35. D. Boyanovsky, I. D. Lawrie, and D.-S. Lee, Phys. Rev. D 54, 4013 (1996).

36. S. Juchem, W. Cassing, and C. Greiner, hep-ph/0307353.

37. W. Cassing and S. Juchem, Nucl. Phys. A 665, 377 (2000); Nucl. Phys. A 672, 417 (2000); Nucl. Phys. A 677, 445 (2000). 